\title{
Paroxysmal nocturnal hemoglobinuria (PNH): higher sensitivity and validity in diagnosis and serial monitoring by flow cytometric analysis of reticulocytes
}

\author{
Britta Höchsmann • Markus Rojewski • \\ Hubert Schrezenmeier
}

Received: 8 July 2010 / Accepted: 31 January 2011 / Published online: 26 February 2011

(C) The Author(s) 2011. This article is published with open access at Springerlink.com

\begin{abstract}
Flow cytometric analysis of GPI-anchored proteins (GPI-AP) is the gold standard for diagnosis of paroxysmal nocturnal hemoglobinuria $(\mathrm{PNH})$. Due to therapy options and the relevance of GPI-deficient clones for prognosis in aplastic anaemia detection of PNH is gaining importance. However, no generally accepted standard has been established. This study analysed the usefulness of a flow cytometric panel with CD58, CD59 on reticulocytes and erythrocytes, CD24/CD66b and CD16, FLAER on granulocytes and CD14, and CD48 on monocytes. Actual cut-off (mean $+2 \mathrm{SD}$ ) for GPI-deficient cells was established in healthy blood donors. We studied 1,296 flow cytometric results of 803 patients. Serial monitoring was analysed during a median follow-up of 1,039 days in 155 patients. Of all, $22 \%$ and $48 \%$ of 155 follow-up patients. showed significant GPI-AP-deficiency at time of initial analyses. During follow-up in 9\%, a new PNH diagnosis, and in $28 \%$, a significant change of size or lineage involvement was demonstrated. Highly significant correlations for GPI-AP deficiency were found within one
\end{abstract}

$\mathrm{BH}$ and HS contributed equally to this manuscript.

Electronic supplementary material The online version of this article (doi:10.1007/s00277-011-1177-4) contains supplementary material, which is available to authorized users.

B. Höchsmann $(\bowtie) \cdot$ H. Schrezenmeier

DRK-Blood Donor Service, Baden-Württemberg-Hessia,

Institute for Clinical Transfusion Medicine

and Immunogenetics Ulm,

Helmholtzstraße 10,

89081 Ulm, Germany

e-mail: b.hoechsmann@blutspende.de

M. Rojewski

Institute of Transfusion Medicine, University of Ulm,

Ulm, Germany cell lineage $\left(r^{2}=0.61-0.95, p<0.0001\right)$ and between the different cell lineages $\left(r^{2}=0.49-0.88, p<0.0001\right)$. Especially for detection of small GPI-deficient populations, reticulocytes and monocytes proved to be sensitive diagnostic tools. Our data showed superiority of reticulocyte analyses compared with erythrocyte analyses due to transfusion and hemolysis independency especially in cases with small GPI-deficient populations. In conclusion, a screening panel of at least two different GPI-AP markers on granulocytes, erythrocytes, and reticulocytes provides a simple and rapid method to detect even small GPI-deficient populations. Among the markers in our panel, CD58 and CD59 on reticulocytes, CD24/66b, and eventually FLAER on granulocytes as well as CD14 on monocytes were most effective for flow cytometric diagnosis of GPI deficiency.

Keywords PNH - Paroxysmal nocturnal hemoglobinuria • Flow cytometry $\cdot$ Immunophenotyping .

Glycosylphophatidyl-inositol anchor - GPI deficiency .

Reticulocytes

\section{Introduction}

Paroxysmal nocturnal hemoglobinuria (PNH) is an acquired hematopoietic stem cell disease $[1,2]$. Pathophysiologically, it is based on somatic mutations of the X-linked phosphatidyl-inositol-glycan gene $[3,4]$ which result in a partial or absolute deficiency of GPI-linked proteins [5]. The lack of GPI-linked proteins leads to the clinical features chronic intravascular hemolytic anaemia and thromboembolism [31, 32]. The role of GPI-AP in bone marrow failure which is often associated with PNH is less clear [6].

Measurement of GPI-anchored proteins expression by flow cytometry is nowadays the gold standard for labora- 
tory diagnosis of PNH and has replaced the Ham test and the sucrose lysis test (sugar water test) [7, 8]. Flow cytometry allows a sensitive and specific detection of even small GPI-deficient populations and a quantification of $\mathrm{PNH}$ cell populations in various cell lineages. This quantification is especially important, as the estimated size and type of the involved cell lineages correlates with different clinical manifestations of the disease [14]. For instance, patients with more than $20 \%$ type III deficiency on red cells mostly have clinically significant hemolysis, while nearly half of patients with more than $50 \%$ GPI deficiency on the granulocytes suffer from venous thrombosis in the first 10 years after initial diagnosis [15]. Therefore, the size of the GPI-AP-deficient population on granulocytes is a prognostic factor for vascular events, which have been shown to be the key determinant of prognosis with the most important impact on life expectancy. Remarkably, clone size and cell lineage involvement can change over time $[6,12,21]$. This leads to the need of follow-up investigations to initiate appropriate therapeutic approaches. Beside initial diagnosis, flow cytometry in particular, enables serial monitoring during the course of the disease [9-12]. This allows ontime determination of therapeutic decisions like initiation of prophylactic anticoagulation [15] or application of the C5-antibody eculizumab, which has already proven to reduce hemolytic activity and thromboembolic events [16-19]. Additionally, therapeutic effects may be monitored via flow cytometric analysis of GPI deficiency. Disappearance of the GPIdeficient populations indicates effective therapy after stem cell transplantation, while increase of the GPI-deficient erythrocyte populations upon eculizumab therapy is presumable due to a decrease of hemolytic activity with the result of a prolonged red blood cell (RBC) survival [18]. Although flow cytometric analysis of GPI-AP is the gold standard for diagnosis of PNH for years, there are no detailed international or even nationwide rules to determine minimal demands for PNH diagnosis based on flow cytometry. There exists just a general advice of the International PNH Interest Group (IPIG) to examine at least erythrocytes and granulocytes for diagnosis of PNH [21]. No consensus recommendations are available for the choice of GPI-AP markers or even the cell populations which should be examined. Thus, results from different laboratories are often difficult to compare and falsenegative or false-positive results are not a rare event. Moreover, most tests are influenced by hemolytic crises or RBC transfusions as they rely on GPI deficiency of erythrocytes. Therefore, in this study, we retrospectively analysed the results of our routine diagnostics of the last 5 years to assess the most suitable GPI markers and cell populations for a sensitive, valid, and rapid GPI-AP flow cytometric analysis for diagnosis and serial testing.

\section{Design and methods}

\section{Patients}

Flow cytometric GPI-AP analysis was performed in 803 patients suffering from PNH or suspected GPI-deficient populations. The reason for flow cytometric analysis as reported by the treating physician were the following diagnoses or suspected diagnoses: PNH in 67 patients, hemolysis in 77 patients, aplastic anaemia or trilineage cytopenia in 291 patients, MDS in 44 patients, single lineage or bilineage cytopenia in 76 patients, unclear thromboembolic events or ischemias in 49 patients, other hematologic diseases in 25 patients, and insufficient information in 174 patients. In the majority of the patients, blood was sent for routine diagnostic assessment to our reference diagnostic laboratory at the Institute of Clinical Transfusion Medicine and Immunogenetics Ulm for analysis of GPI deficiency as a diagnostic routine procedure in patients with bone marrow failure, hemolytic anaemia, iron deficiency anaemia, thrombosis, or abdominal pain of unknown origin. Some of the analyses were performed as diagnostic test in clinical trials after informed consent and ethical committee approval of the trial. Patients were included in the follow-up analysis if at least two GPI-AP assessments with a minimum interval of 3 months were available ( $n=155$ patients). At time of initial GPI-AP analysis, blood count was available in 89 of the 155 followup patients; in this patients group, $48 / 89$ patients showed neutropenia with $<0.5 \mathrm{G} / 1$ and $70 / 89$ patients had thrombopenia with $<100$ G/l. Patients with eculizumab treatment were censored at the date of first eculizumab application, and GPI-AP assessment of these patients during eculizumab therapy were not included in the analyses presented here. Five patients who received allogeneic stem cell transplantation were not censored for analysis.

The pattern of lineage involvement and the suitability of various lineages and markers in the peripheral blood for detection of GPI-deficient populations were statistical analysed retrospectively in all patients. For follow-up, the size of GPI-deficient populations as well as the evolution of lineage involvement was investigated.

\section{Two-color flow cytometry}

Immunophenotyping was performed as previously described [12]. Flow cytometric examinations $(1,296)$ performed between March 2003 and August 2008 were analysed. Peripheral blood (PB) was obtained by venous puncture using Na-EDTA or citrate as anti-coagulant. All incubations were performed at room temperature in the dark. We used the flow cytometric analysis of CD58 and CD59 on reticulocytes and erythrocytes and of CD66b/ CD24 and CD16 on granulocytes as mandatory markers for 
our screening panel. The expressions of CD14 and CD48 on monocytes as well as CD48/CD19 and CD48/CD3 on lymphocytes were used as optional markers to fully assess lineage involvement. In 584 cases, a combination of CD66b/CD24, CD16, and FLAER [23], a fluorochromelabelled modified bacterial toxin which binds directly to the GPI anchor was used to confirm the diagnosis. Staining of the reticulocytes by the RNA dye thiazol orange allowed a separate analysis of erythrocytes and reticulocytes.

Staining of erythrocytes and reticulocytes (no wash, stain, no lyse protocol)

A volume of $100 \mu \mathrm{L}$ of $1: 10$ diluted PB in PBS without $\mathrm{Ca}^{2+} / \mathrm{Mg}^{2+}$ (PAA Laboratories, Paching, Austria) was used for each assay. As isotype control, diluted $\mathrm{PB}$ was incubated with $20 \mu \mathrm{L}$ of $\mathrm{IgG}^{*} \mathrm{PE}$, clone $\times 40$ (BD Immunocytometry Systems, Heidelberg, Germany). De- tection of GPI-deficient erythrocytes and reticulocytes was performed by staining $100 \mu \mathrm{L}$ of diluted $\mathrm{PB}$ with either $20 \mu \mathrm{L}$ of CD58*PE, clone AICD58 (Beckman Coulter Immunotech, Krefeld, Germany) or $20 \mu \mathrm{L}$ of CD*59 PE, clone p282 (H19) (BD Pharmingen, Heidelberg, Germany). After $20 \mathrm{~min}$, cells were washed once by addition of $1 \mathrm{~mL}$ PBS without $\mathrm{Ca}^{2+} / \mathrm{Mg}^{2+}$ followed by centrifugation at 5,900 $\times \mathrm{g}$ for $0.5-1 \mathrm{~min}$. The cells of all three assays were resuspended in $1 \mathrm{~mL}$ of ReticCOUNT Reagent (BD Immunocytometry Systems) and incubated for $20 \mathrm{~min}-1 \mathrm{~h}$ to stain RNA of reticulocytes before analysis by flow cytometry (Fig. 1).

Staining of granulocytes, monocytes (no wash, stain, lyse protocol)

To consider the contribution of different autofluorescences, separate isotype controls were performed for granulocytes

\section{healthy control}

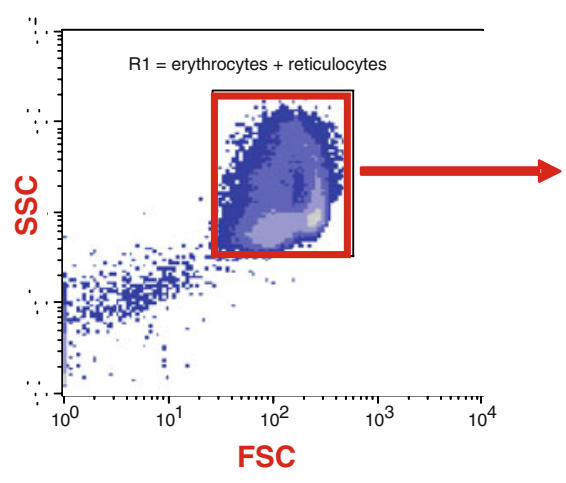

erythrocyte/reticulocyte-gate

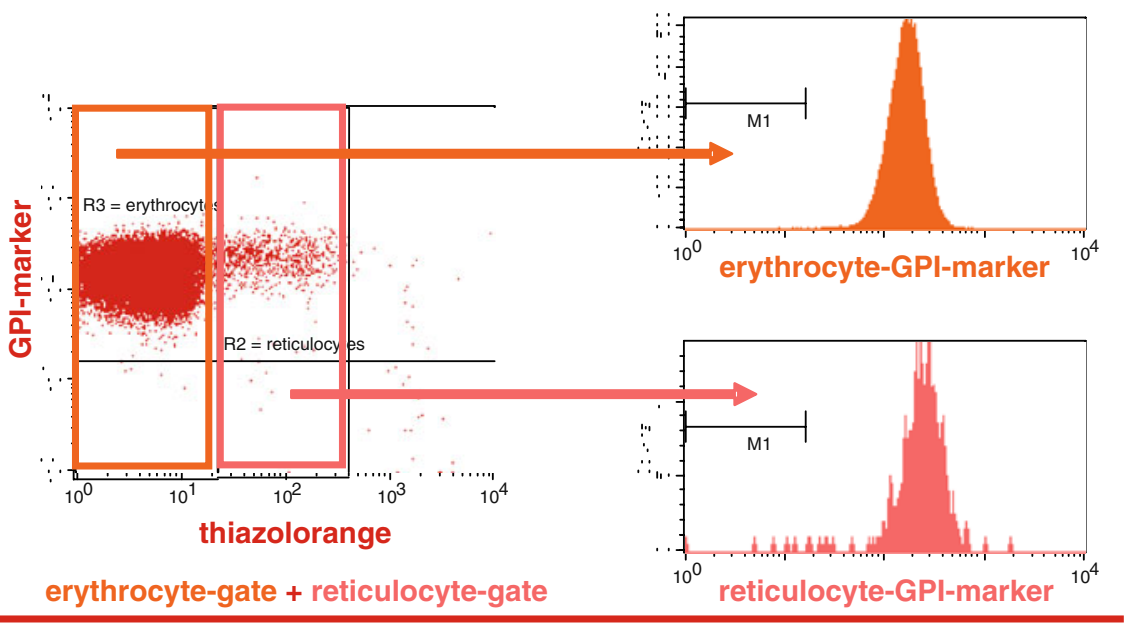

\section{PNH-patient}

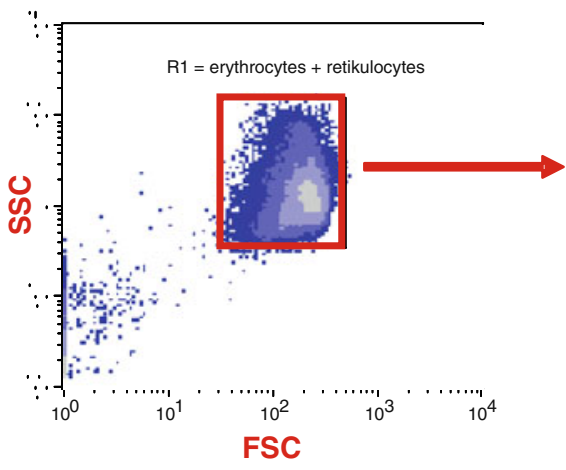

erythrocyte/reticulocyte-gate

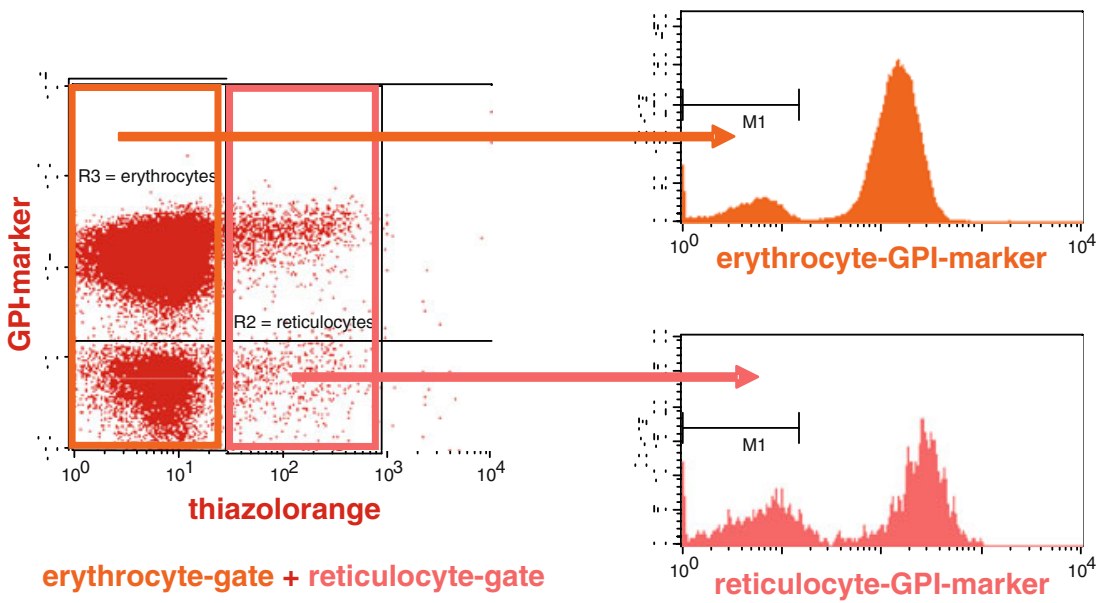

erythrocyte-gate + reticulocyte-gate quadrant) without significant GPI deficiency. In the PNH patient, separation of four populations according to erythrocytes and reticulocytes without (upper quadrants) and with (lower) significant GPI deficiency the healthy control, separation of two populations according to erythrocytes (left upper quadrant) and reticulocytes (right upper 
and monocytes. Therefore, two assays of $\mathrm{PB}$, containing $0.8 \times 10^{6}-1.6 \times 10^{6}$ leukocytes each, were incubated with $20 \mu \mathrm{L}$ of IgG*FITC and $20 \mu \mathrm{L}$ of IgG* PE clone $\times 40$ (both BD Immunocytometry Systems). Analysis of granulocytes was performed by staining PB with $10 \mu \mathrm{L}$ of CD24*FITC, clone SN3 (DAKO, Hamburg, Germany), $10 \mu \mathrm{L}$ of CD66b*FITC, clone 80H3 (Beckman Coulter Immunotech), and $20 \mu \mathrm{L}$ of CD11b*PE, clone D12 (BD Immunocytometry Systems). In a separate assay, the corresponding amount of PB was incubated with $10 \mu \mathrm{L}$ of CD16*FITC, clone NKP15 and $20 \mu \mathrm{L}$ of CD11b*PE, clone D12 (both BD Immunocytometry Systems). For analysis of monocytes, PB was incubated either with $20 \mu \mathrm{L}$ of CD14*FITC, clone MOP9 and $20 \mu \mathrm{L}$ of $\mathrm{CD} 33 * \mathrm{PE}$, clone P67.6 (both BD Immunocytometry Systems) or $20 \mu \mathrm{L}$ of CD48*FITC, clone J4.57 (Beckman Coulter Immunotech) and $20 \mu \mathrm{L}^{*} \mathrm{CD} 33$, clone P67.6 (BD Immunocytometry Systems), respectively. After 20-30 min, cells were washed once by addition of $1 \mathrm{~mL}$ PBS without $\mathrm{Ca}^{2+} / \mathrm{Mg}^{2+}$ and centrifugation by $5,900 \times g$ for $0.5-1 \mathrm{~min}$. Lysis of erythrocytes was performed by resuspension of the cell pellets in $100 \mu \mathrm{L}$ OptiLyse B (Beckman Coulter Immunotech), incubation for 7-15 min, addition of $1 \mathrm{~mL}$ aqua followed by vigorous mixing, and incubation for another 5-15 $\mathrm{min}$. After lysis of erythrocytes, cells were washed twice using $1 \mathrm{~mL}$ PBS without $\mathrm{Ca}^{2+} / \mathrm{Mg}^{2+}$ and resuspended $300-$ $800 \mu \mathrm{L}$ PBS without $\mathrm{Ca}^{2+} / \mathrm{Mg}^{2+}$ for flow cytometry analysis.

\section{Staining of granulocytes (wash, lyse, stain protocol)}

For detection of GPI-deficient granulocytes by FLAER (FITC labeled aerolysin, Protox Biotech, Victoria, Canada), a volume of PB blood containing approximately $2 \times 10^{5}$ neutrophils was washed with the fivefold volume of PBS without $\mathrm{Ca}^{2+} / \mathrm{Mg}^{2+}$, and the supernatant was discarded. Erythrocytes were lysed as described above using half the volume of OptiLyse B and aqua and washed twice, using PBS without $\mathrm{Ca}^{2+} / \mathrm{Mg}^{2+}$. Half of the remaining cells were stained with $2-5 \mu \mathrm{L}$ of a $1: 10$ dilution of $\operatorname{IgG}^{*} \mathrm{PE}$, clone $\times 40$ (BD Immunocytometry Systems) in PBS, the other half of the cells was stained with a 1:50 dilution of CD16*PE, clone 3G8 (BD Pharmingen) in PBS and 0.5-5 $\mu$ LLAER, respectively. After 20-30 min, $1 \mathrm{~mL}$ of PBS without $\mathrm{Ca}^{2+}$ / $\mathrm{Mg}^{2+}$ was added, supernatant was removed after centrifugation at $5,900 \times \mathrm{g}$ for $30 \mathrm{~s}-1 \mathrm{~min}$, and cells were resuspended in $300-800 \mu \mathrm{L}$ of PBS without $\mathrm{Ca}^{2+} / \mathrm{Mg}^{2+}$.

Normal range - cut off values of GPI deficiency

In the 5-year observation period every week, healthy blood donors (overall $n=268$ ) were tested for GPI deficiency to establish cut-off values (mean+2 SD) for GPI-deficient cells. In respect of variabilities in antibody efficiency, we updated this cut-off every 6 months with the results of the last 50-60 examined healthy donors. The valid cut-offs at the end of the 5-year observation period (overall results of the 268 healthy donors were put in parentheses) were: reticulocytes CD58, 0.5\% (1.0\%), reticulocytes CD 59, $1.9 \%(2.0 \%)$, erythrocytes CD $58,0.1 \%(0.2 \%)$, erythrocytes CD59, $0.5 \%(0.8 \%)$, granulocytes CD66b/CD24, $0.1 \%(0.7 \%)$, granulocytes CD16, $1.0 \%(1.2 \%)$, granulocytes FLAER*,CD16, $0.3 \%$ (1.2\%); monocytes CD14, $2.5 \%$ (3.3\%), monocytes CD $48,1.1 \%$ (1.1\%).

For flow cytometric diagnosis of a PNH cell population, at least two cell lineages with significant GPI-deficient populations were required. Significant GPI-deficient populations are defined if all examined markers (minimum, 2) were exceeding the cut-off value.

\section{Statistical analysis}

Statistical analyses of the data were carried out in Microsoft Office Access 2003 and GraphPad PRISM, version 4.00. Used tests were linear regression, including correlations, coefficient of correlation, and confidence interval as well as scatter plots. A $p$ value of less than 0.05 was considered as statistically significant.

\section{Results}

Patient characteristics

Patient characteristics for the examined 803 patients (416 male; 382 female) were as follows: the patient age ranged from 0.4 to 90.7 years (median age, 32.4 years) with no relevant differences between men (52\%) and women (48\%) (Table 1). At initial GPI-AP flow cytometry, 176 of all patients (22\%) were diagnosed with PNH typical GPIdeficient populations (Fig. 2). The groups with and without PNH diagnosis at time of initial GPI-AP analysis do not differ significantly in age and sex distribution. For detailed patient characteristics, see Table 1 .

In the follow-up cohort, 155 patients and 625 flow cytometric analyses were evaluated (median number of analyses per patient, 3; range from 2 to 26) for a median follow-up duration of 1,039 days. Patient characteristics of the follow-up group are summarised in Table 1.

Blood count was evaluable in 89 of these patients at the time of initial assessment and in 120 patients at time of the last examination. Thrombocytopenia $<100 \mathrm{G} / 1$ was obtained in $62 \%$ at the initial examination and in $43 \%$ at the last examination. Leukocytopenia $<0.5 \mathrm{G} / 1$ was initially obtained in $36 \%$ and decreased to $18 \%$ at the final investigation (see data supplements). 


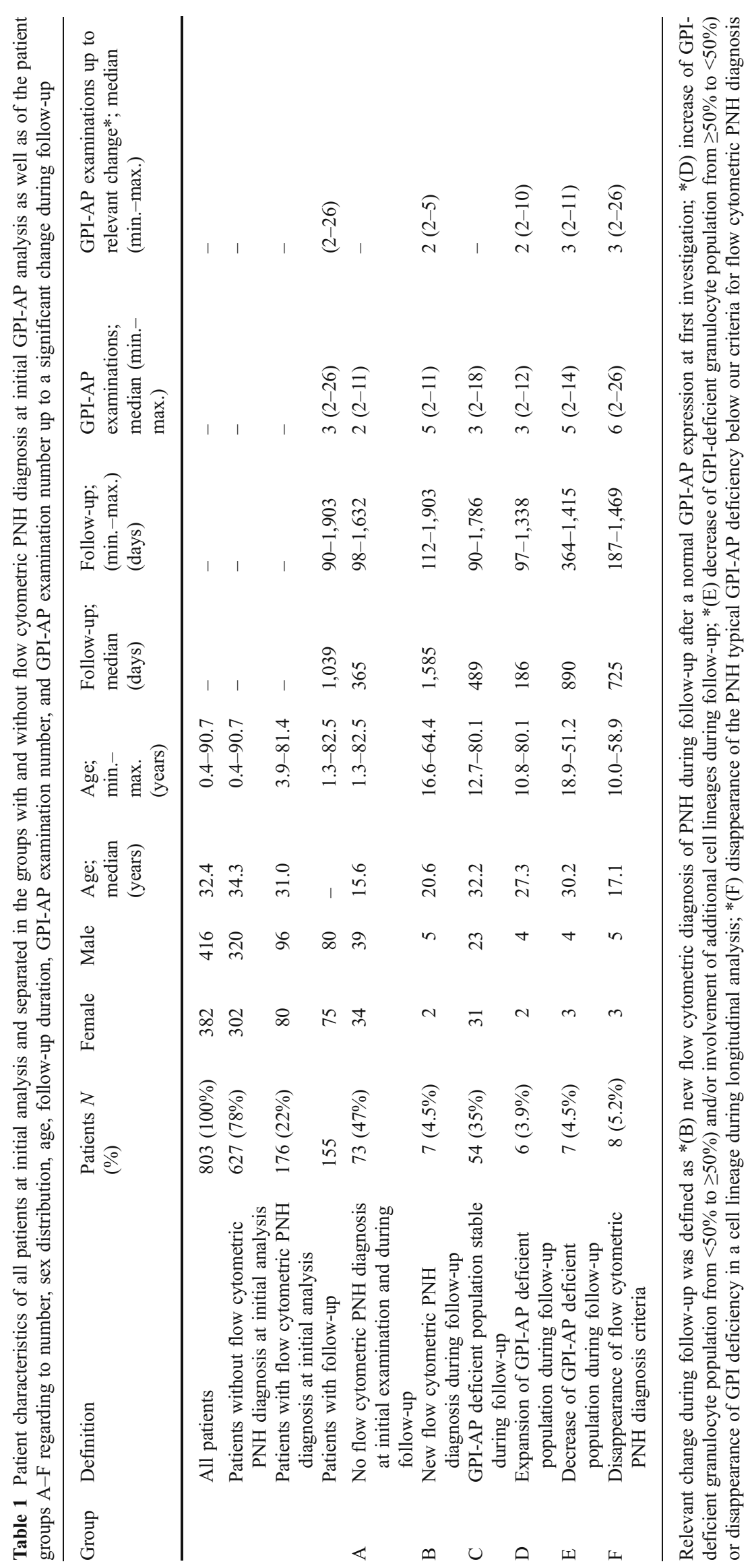




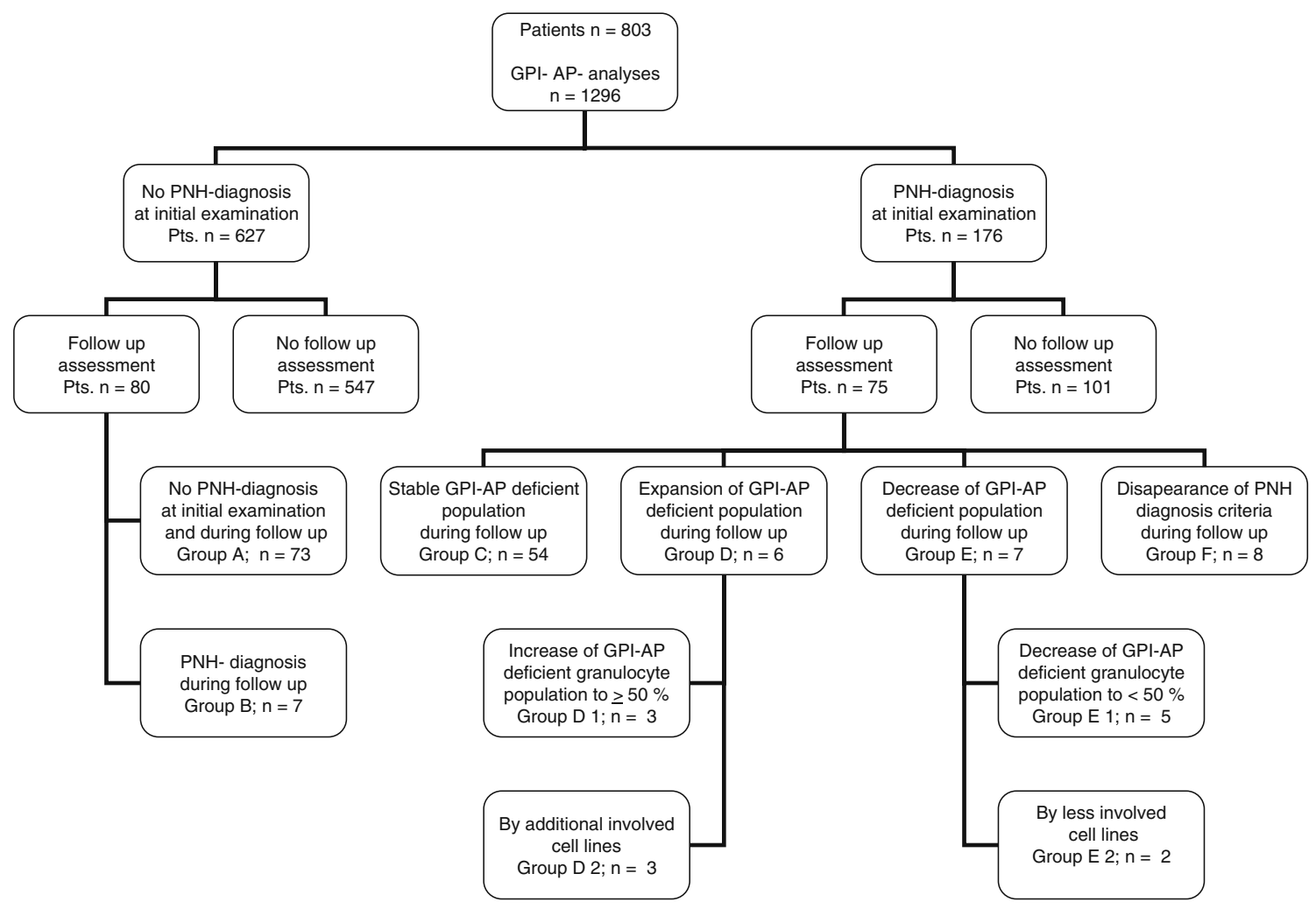

Fig. 2 Flow sheet patient of the different patient groups at time of initial GPI-AP analysis and during follow-up. Significant changes of GPI-deficient populations during follow-up was observed in groups B, D1, D2 with an expansion of GPI-AP-deficient populations (including

Flow cytometric results

\section{Analysis of different GPI-anchored markers in the same cell lineage}

Primarily, we compared all flow cytometric results (including the follow-up analyses) within each separate cell lineage to assess the correlation of the various GPI-linked markers. We found a highly significant correlation between the markers CD58 and CD59 on reticulocytes $\left(r^{2}=0.95\right.$, $p<0.0001 ; n=1,284)$ and erythrocytes $\left(r^{2}=0.86, p<\right.$ $0.0001 ; n=1,284)$, between $\mathrm{CD} 24 / 66 \mathrm{~b}$ and $\mathrm{CD} 16$ or between CD24/66b and CD16, FLAER on granulocytes $\left(r^{2}=0.76, p<0.0001 ; n=1248\right)$, and between CD14 and CD48 on monocytes $\left(r^{2}=0.62, p<0.0001 ; n=599\right)$ (Fig. 3). If we restricted the analysis to the first GPI-AP flow cytometric analysis in each patient, the correlation did not change considerably (Fig. 3).

\section{Marker analysis between different cell lineages}

In a second step, we compared the sizes of various GPImarker negative populations in different cell lineages. This analysis was initially performed for all GPI-AP flow new PNH diagnosis) and in groups E1, E2, and F with a decrease of GPI-AP-deficient populations (including disappearance of $\mathrm{PNH}$ diagnosis criteria)

cytometric results in patients with flow cytometric diagnosis of PNH. The following significant correlations were obtained: CD58-negative reticulocytes and CD58-negative erythrocytes $\left(n=457, r^{2}=0.63, p<0.0001\right)$, CD59-negative reticulocytes and CD59-negative erythrocytes $\left(n=455, r^{2}=\right.$ 0.61, $p<0.0001)$, CD59-negative reticulocytes and CD24/ D66b-negative granulocytes $\left(n=450, r^{2}=0.60, p<0.0001\right)$, CD59-negative reticulocytes and CD14-negative monocytes $\left(n=381, r^{2}=0.68, p<0.0001\right)$, CD59-negative erythrocytes and CD24/66b-negative granulocytes $\left(n=449, r^{2}=\right.$ 0.33, $p<0.0001)$, CD14-negative monocytes and CD24/ 66b-negative granulocytes $\left(n=423, r^{2}=0.84, p<0.0001\right)$, CD48-negative monocytes and CD24/66b-negative granulocytes $\left(n=421, r^{2}=0.48, p<0.0001\right)$ (Fig. 4).

In a second step, the analysis was restricted to the first flow cytometric analysis of each patient with flow cytometric diagnosis of PNH. There were no significant differences between both analyses (Fig. 4).

\section{Marker analysis for detection of small GPI-deficient populations}

To elucidate the importance of individual markers for the flow cytometric diagnosis of $\mathrm{PNH}$, all markers were 
Fig. 3 Correlation between the used GPI-AP markers within one cell line. Black=all analyses. $R e d=$ restricted to the first analysis per patient. Broken black lines $=$ confidence interval of all analyses. Broken red lines $=$ confidence interval of the first analysis per patient. All correlations were highly significant ( $p$ $<0.0001$ ) in the group with all analyses as well as in the group with only the first analysis ( $p<$ $0.0001)$. The range of the correlation coefficient was $r^{2}=$ 0.61-0.95
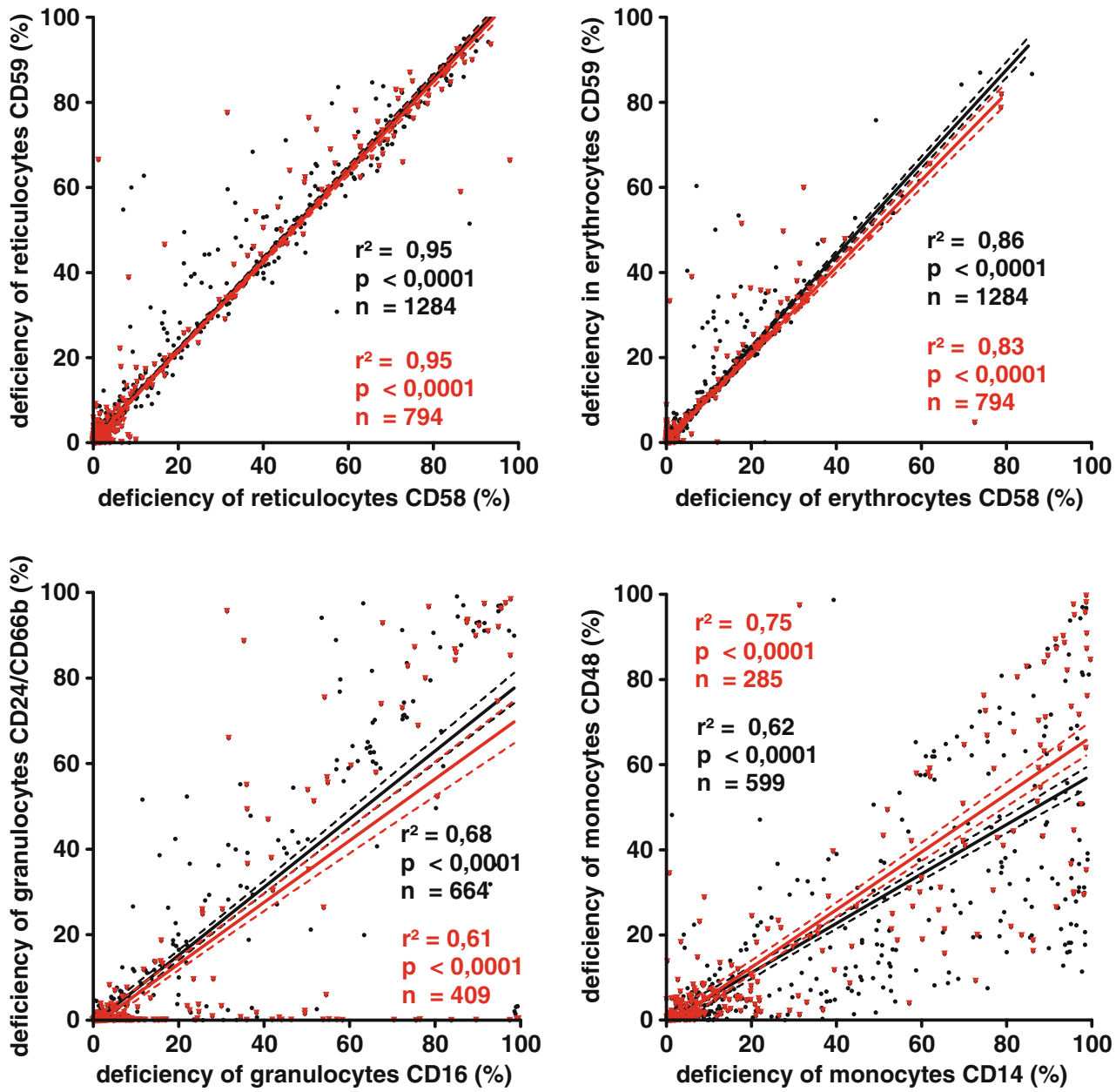

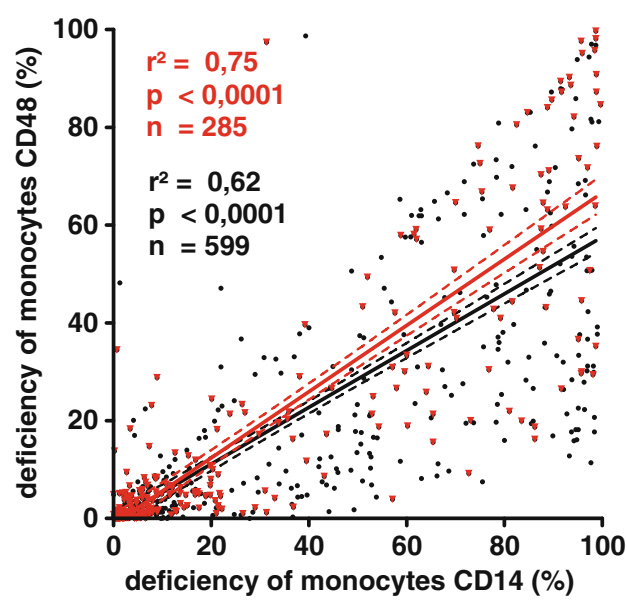

separately analysed. Depending on the size of the CD24/ CD66b-negative granulocyte population, we defined two different subgroups based on a CD24/CD66b-negativegranulocyte population $<10 \%$ or $\geq 10 \%$ at the first investigation. The largest median GPI-deficient population in the subgroup with $<10 \%$ CD24/66b-negative granulocyte populations was observed for CD14 on monocytes, CD16 or CD16, FLAER on granulocytes, and CD59 on reticulocytes. In the subgroup, with $\geq 10 \%$ CD24/CD66b-negativegranulocyte population, the largest median GPI-deficient population was shown for CD14 on monocytes, CD24/66b and CD16 as well as CD16, FLAER on granulocytes, and CD59 on reticulocytes (Fig. 5).

\section{Follow-up analysis}

We analysed repeated measurements of GPI-AP expression on reticulocytes, erythrocytes, granulocytes, and monocytes in 155 patients (Fig. 6) in order to study the dynamics of GPI-AP deficient cell populations over time. Since it has been demonstrated that treatment with eculizumab changes, the relative proportion of GPI-AP deficient red cells [19], patients who received eculizumab treatment were censored at start of this treatment. Median number of analyses per patient was 3 and the median follow-up duration was 1,039 days (minimum 90 days, maximum 1,903 days). Initially, $75(48 \%)$ of these patients were diagnosed with PNH. The longitudinal studies revealed different patterns of GPI-AP expression during the follow-up period (Figs. 2, 6, and 7): (A) no significant GPI-AP deficiency fulfilling our criteria for flow cytometric $\mathrm{PNH}$ diagnosis during the whole investigation period; (B) new flow cytometric diagnosis of PNH during follow-up after a normal GPIAP expression at first investigation; (C) flow cytometric diagnosis of PNH at initial analysis with stable size of GPIdeficient granulocyte population during follow-up (defined as GPI-deficient granulocyte population stable $<50 \%$ or $\geq 50 \%$ ); (D) flow cytometric diagnosis of $\mathrm{PNH}$ at initial analysis with expansion of the GPI-deficient granulocyte population (defined as increase of GPI-deficient granulocyte population from $<50 \%$ to $\geq 50 \%$ ) (D1) and/or involvement of additional cell lineages (D2) during follow-up; (E) flow cytometric diagnosis of PNH at initial analysis and decrease of the GPIdeficient granulocyte population (defined as decrease of GPIdeficient granulocyte population from $\geq 50 \%$ to $<50 \%$ ); (E1) or disappearance of GPI deficiency in a cell lineage (E2) 

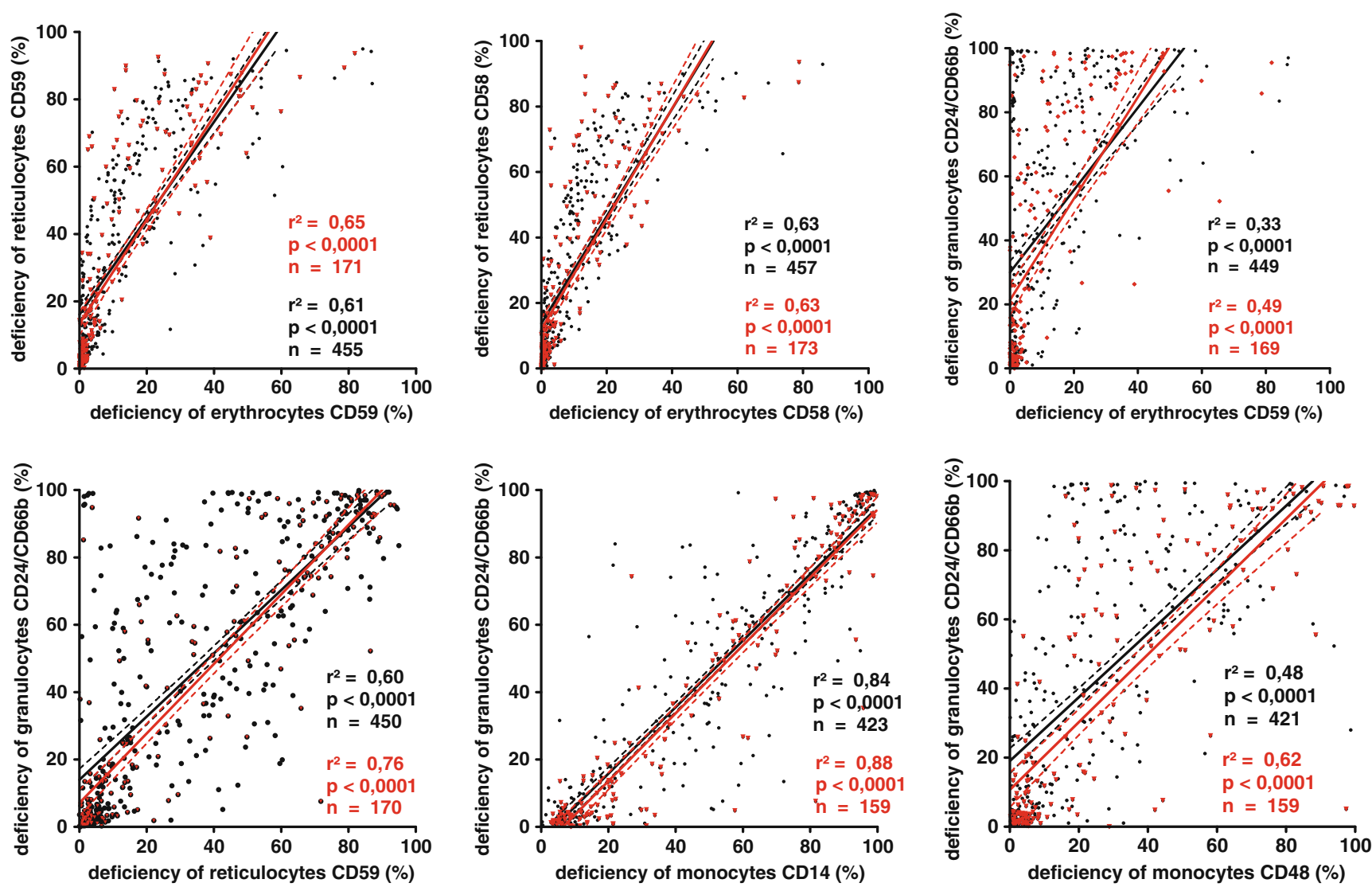

Fig. 4 Correlation between the used GPI-AP markers on the different cell lines. Black $=$ all analyses. Red $=$ restricted to the first analysis per patient. Broken black lines $=$ confidence interval of all analyses. Broken red lines $=$ confidence interval of the first analysis per patient. All correlations were highly significant in the group with all analyses $(p<$ $0.0001)$ as well as in the group with only the first analysis $(p<0.0001)$.
The range of the correlation coefficient was $r^{2}=0.33$ up to 0.88 . The lowest value for $r^{2}\left(r^{2}=0.33\right)$ was observed between CD59 on erythrocytes and CD24/CD66b on granulocytes, whereas the correlation between CD59 on reticulocytes and CD24/CD66b on granulocytes was $r^{2}=0.60$ during longitudinal analysis; (F) disappearance of the PNH typical GPI-AP deficiency below our criteria for flow cytometric PNH diagnosis. The cut-off granulocyte clone size of $<1 \geq 50 \%$ as sign of a relevant quantitative change in clone size was chosen because of published data about the impact of this relative proportion for thromboembolic events [15]. Patient characteristics, number of examinations, and duration of follow-up are summarised in Table 1. In detail, 9\% of the 80 patients with no PNH typical GPI-AP deficiency in the first analysis developed flow cytometric $\mathrm{PNH}$ diagnosis during follow-up (group B). In three of these seven cases detected during follow-up, diagnosis was based on significant GPI-AP-deficient populations on monocytes and granulocytes. Of the follow-up patients, $28 \%$ showed a significant change of GPI-AP deficiency on granulocytes or lineage involvement. Moreover, 5 out of the 75 patients initially diagnosed with PNH developed an emerging GPIdeficient population on an additional cell lineage or showed less involved cell lineages during follow-up (group D2 and
E2). In $11 \%$ of all cases with flow cytometric diagnosis of $\mathrm{PNH}$, a significant change of GPI-deficient granulocyte population occurred (group D1 and E1). In the group of the seen patients with decrease of GPI-deficient granulocyte population or disappearance of significant GPI-AP deficiency in a cell line (E1 and E2), two patients received allogeneic stem cell transplantation, and two patients were treated with ATG-based immunosuppressive therapy. Only three of these seven patients suffered from a classical $\mathrm{PNH}$, while four were diagnosed with associated bone marrow failure syndromes. All three patients with classical PNH showed a decrease of GPI-AP deficient population on granulocytes and no change in cell lineage involvement. Additionally, eight patients (11\%) fulfilled no longer the flow cytometric criteria of PNH during follow-up (group F). Half of these eight patients received an ATG-based immunosuppression and four patients (one was treated for MDS) received an allogeneic stem cell transplantation before the PNH typical GPI-AP deficient population pattern disappeared. A sponta- 

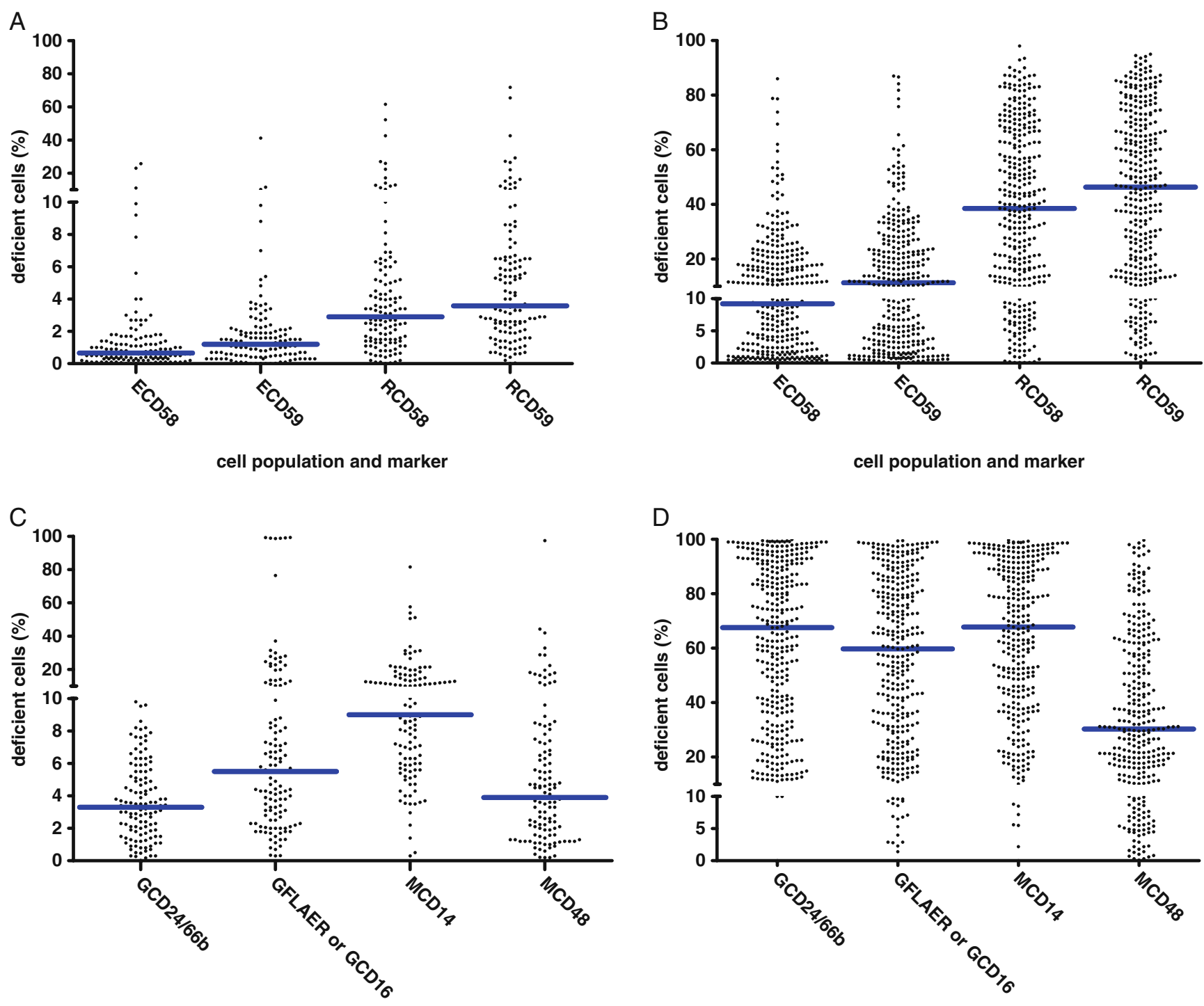

cell population and marker

Fig. 5 Scatter blots with marker deficient cells in percent in patients with $\geq 10 \%$ GPI-deficient granulocyte population $(B+D)$ and in patients with $<10 \%$ GPI-deficient granulocyte population $(\mathrm{A}+\mathrm{C})$. ECD58 $=$ CD58-deficiency on erythrocytes, ECD59 $=$ CD59 deficiency on erythrocytes, $\mathrm{RCD} 58=\mathrm{CD} 58$ deficiency on reticulocytes, $\mathrm{RCD} 59=$

neous loss of significant GPI deficiency clones, i.e., disappearance without prior therapy, was observed in none of the follow-up patients. All such cases were obtained in association with therapy response and blood count improvement.

\section{Discussion}

Flow cytometry of GPI-AP is considered to be the gold standard of PNH diagnosis. CD55 and CD59 expression on erythrocytes are most frequently used for screening of PNH $[6,7,9-14,21,22]$. Our presented data show that addition

on reticulocytes, GCD24/66b $=\mathrm{CD} 24 / 66 \mathrm{~b}$-deficiency on granulocytes, GFLAER or GCD16=CD16 and/or FLAER or CD16-deficiency on granulocytes, MCD14=CD14-deficiency on monocytes, MCD48= CD48 deficiency on monocytes. Blue line median size of GPIdeficient population

of further antibodies against GPI-linked antigens on different cell lineages considerably improves the sensitivity and validity of the method. Among the markers evaluated, we recommend a panel including at least the following markers: CD58 and CD59 for reticulocytes and erythrocytes a combination of CD24/66b and eventually FLAER on the granulocytes and CD14 on monocytes.

It has been shown that detection of GPI-deficient reticulocytes is more sensitive than analysis of erythrocytes, and reticulocytes better correlates with proportion of GPIdeficient white cells. The remarkable worse correlation between erythrocytes and granulocytes compared to the correlation of reticulocytes and granulocytes (Fig. 4) and 
Follow-up group A: Pat. UL-0180

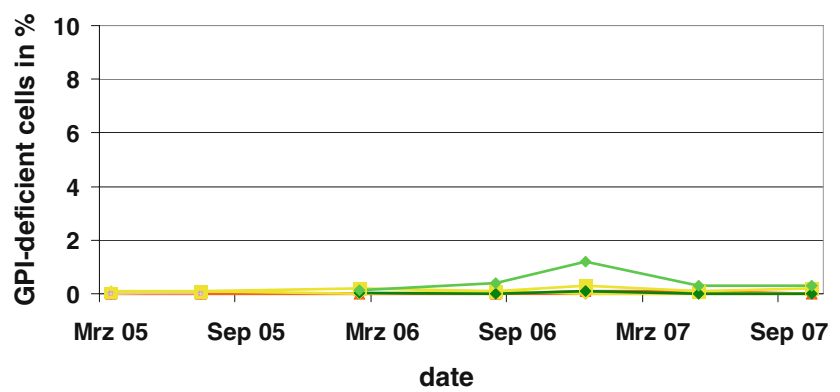

C

Follow-up group C: UL-0026

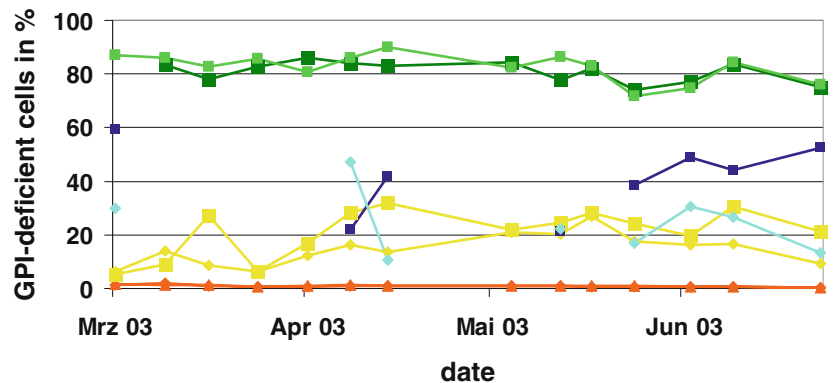

$E$

Follow-up group E: UL-0158

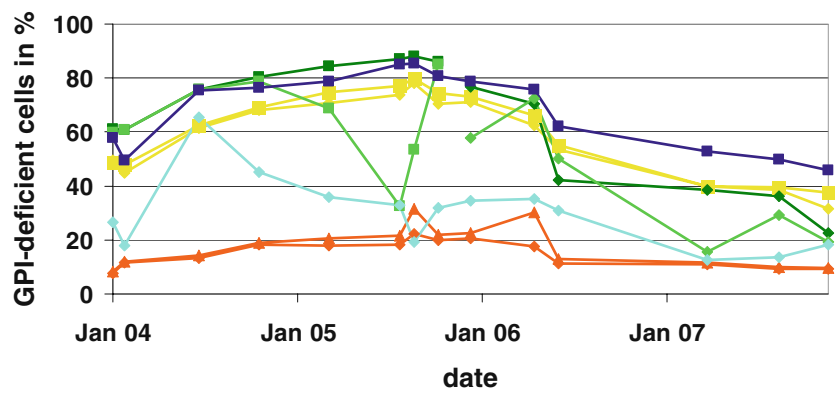

- Reticulocytes CD58,

- Granulocytes CD16,

Granulocytes CD24_CD66b,

- Reticulocytes CD59,
- Granulocytes FLAER/CD16,
- - Monocytes CD14,

Fig. 6 Examples of follow-up courses for each follow-up subgroup A-F. a Flow cytometric criteria for PNH at initial diagnosis and during follow-up not fulfilled; b New flow cytometric diagnosis of PNH during follow-up. c Stable GPI deficiency during follow-up. d

the larger GPI-deficient populations in reticulocyte than erythrocyte (Fig. 5) are probably caused by RBC transfusions, hemolytic crises, and a shorter life span of PNH erythrocytes [24, 25]. As reticulocytes are less affected by these events, their examination for GPI-AP deficiency is especially suitable for diagnosis of involvement of the red cell lineage if there are small GPI-deficient granulocyte populations below $10 \%$. Nevertheless, the most international recommendations for flow cytometry still favour erythrocyte analysis. Our data emphasise the importance of
B

Follow-up group B: Pat. UL-0062

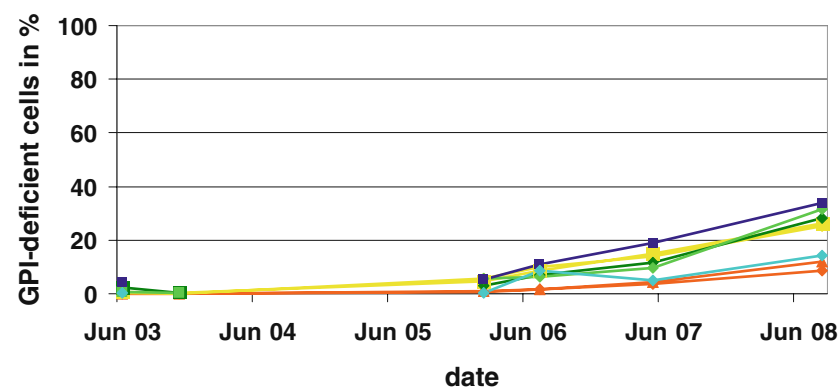

D

Follow-up group D: UL-0255

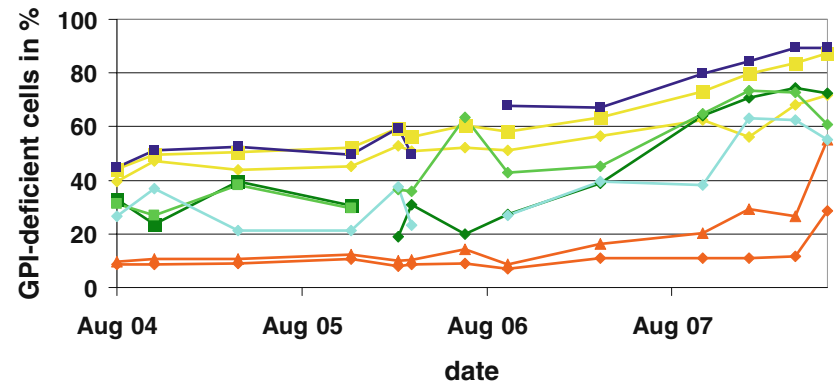

$\mathrm{F}$

Follow-up group F: UL-0006

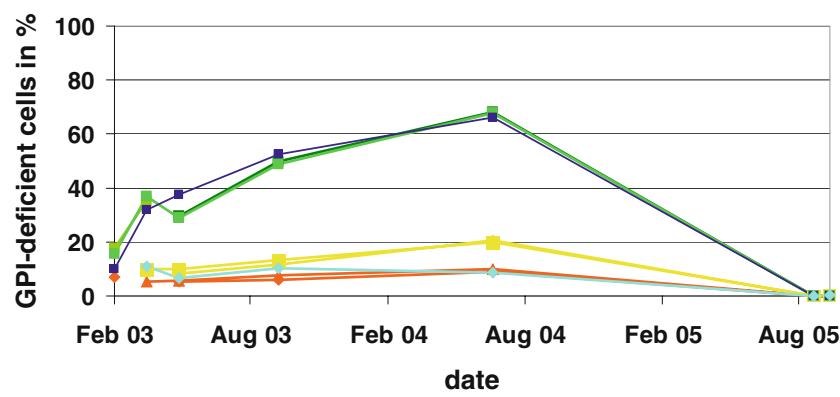

- Erythrocytes CD58,
-- Granulocytes CD16/CD66b,

- Erythrocytes CD59,

* Granulocytes CD24/66b,

Increase of GPI deficiency during follow-up. e Decrease of GPI deficiency during follow-up. f Flow cytometric diagnosis criteria for $\mathrm{PNH}$ lost during follow-up

reticulocyte analysis. Even if reticulocyte clone size correlates well with white cell clone size, both should be tested to confirm the flow cytometric diagnosis of PNH because alteration of GPI-AP on leukocytes can be a sign of granulocytic/monocytic dysplasia and immaturity ([30], data supplements) in the context of various myeloid diseases or cytolysis. Due to our results, participation of leukocytes is detected best by examination of CD14deficiency on monocytes (Fig. 5). Therefore, additional examination of monocytes provides a higher detection 


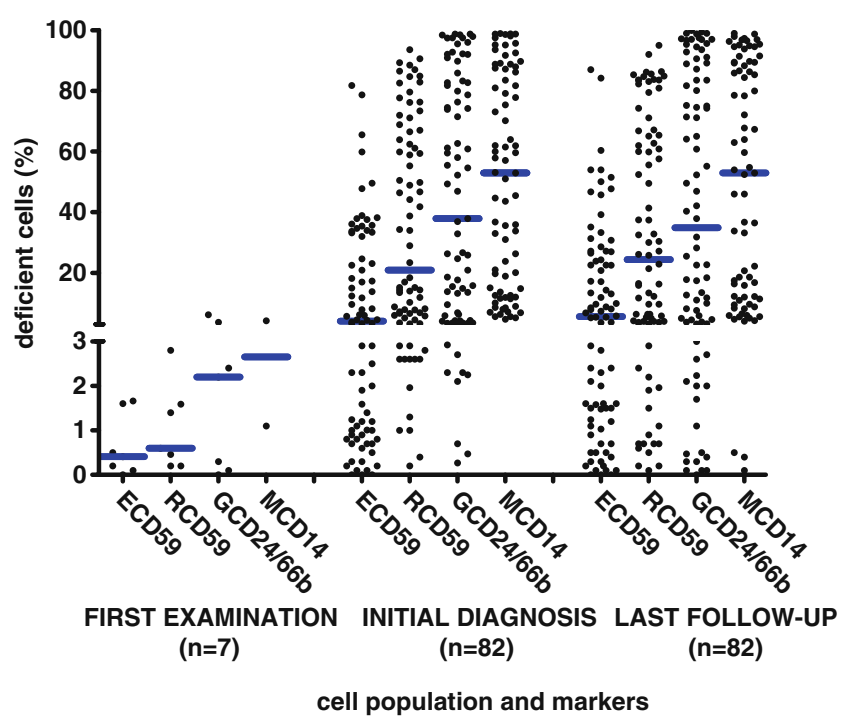

Fig. 7 Scatter blots of GPI-marker deficiency in percent during follow-up. ECD59=CD59 deficiency on erythrocytes, $\mathrm{RCD} 59=$ on reticulocytes, $\mathrm{GCD} 24 / 66 \mathrm{~b}=\mathrm{CD} 24 / 66 \mathrm{~b}$ deficiency on granulocytes, MCD14=CD14 deficiency on monocytes. Blue line: median size of GPI-deficient population. First examination=results of group B (no flow cytometric PNH diagnosis at first GPI-AP-analysis, but fulfilling flow cytometric PNH diagnosis criteria during follow-up) at time of initial GPI-AP analysis. Initial diagnosis $=$ results of group $\mathrm{C}+\mathrm{D}+\mathrm{E}+\mathrm{F}$ (flow cytometric PNH diagnosis at first GPI-AP analysis) at time of first GPI-AP-analysis and results of group B at time of first flow cytometric PNH diagnosis. Last follow-up: results of all patients with flow cytometric PNH diagnosis at time of last GPI-AP analysis

sensitivity of GPI-AP deficiency especially in patients with small GPI-deficient granulocyte populations $<10 \%$. In fact, the marker combination $\mathrm{CD} 24 / 66 \mathrm{~b}$ and CD16 seems to be suitable in sensitivity and validity to detect GPI-deficient granulocytes in our analysis.

Follow-up examination is recommended by the International PNH Interest Group (IPIG) in case of bone marrow failure syndromes [21] even in case of initially normal flow cytometric analysis. Moreover, regular follow-up studies are indicated in case of known PNH to detect substantial changes in GPI-AP-deficient population size or cell lineage involvement, as both situations may prompt therapeutic consequences or may demonstrate effect of therapeutic interventions. Therefore, we evaluated our panel on different cell lineages for longitudinal monitoring of GPIdeficient populations to determine the frequency of significant changes in cell lineage involvement and GPI-deficient population size. Our definition of a relevant change in GPI-deficient population size $(<>>50 \%$ GPI deficiency on granulocytes) is based on data published by Hall et al. [15], demonstrating that patients with a GPI-deficient population on granulocytes $\geq 50 \%$ have a significant higher risk of thromboembolic events. Our results again support the importance of GPI-AP measurement on reticulocytes to obtain results independent of hemolytic crisis and RBC transfusions.
The described flow cytometry panel could be used for serial monitoring during follow-up to detect evolution of GPI-AP deficient populations and to assess therapy effects. Although follow-up investigations were performed only in a minority of patients with initially normal results ( 80 out of 627 patients), in seven of the follow-up patients, PNH was newly diagnosed. Based on our follow-up data with a relevant proportion of newly developing GPI-AP-deficient populations and significant changes in the size of GPI-APdeficient populations, repeated GPI-AP analysis in regular intervals should be performed, like it is recommended by the International PNH Interest Group (IPIG) [21]. Another interesting fact in our follow-up data is the absence of spontaneous remissions of PNH typical GPI-AP-deficient populations which had been reported. A study with 35 patients referred a rate of $15 \%$ spontaneous remissions [6] in PNH patients, but available GPI-AP flow cytometric results and even HAM-test were limited in this study. All observed remissions in our patient group happened in the context of stem cell transplantation or intensified immunosuppressive therapy especially in patients with AA-PNH syndrome. The reported three patients with classical PNH and decrease of the GPI-AP deficient population on granulocytes below $50 \%$ developed an increase over $50 \%$ GPI-deficient cells on the granulocytes during further follow-up.

Generally, expression of GPI-anchored proteins should be analysed in all situations suspicious of PNH and in bone marrow failure syndromes [20]. According to our data, measurement of at least two different GPI-anchored proteins on granulocytes, erythrocytes, and reticulocytes provides a simple and rapid method to detect even small GPI-deficient populations. Measurement of erythrocytes only includes the pitfall of false-negative results or false low values of GPI-deficient populations. On the contrary, examination of GPI deficiency on reticulocytes additionally prevents false positive interpretation of significant GPIdeficient populations on the leukocytes (data supplements). GPI-AP deficiencies without clinical PNH were reported in the context of other hematologic diseases or after immunosuppressive therapy [26] as well as in healthy individuals $[27,28]$. Our data demonstrate that in patients with small GPI-deficient populations, these can in particular be detected on monocytes. In conclusion, we recommend a flow cytometric screening panel with the markers CD58 and CD59 on reticulocytes and erythrocytes as well as with the markers CD24/66b and CD16 on granulocytes for initial diagnosis and monitoring during follow-up. In case of significant GPI-AP-deficient population in a minimum of one cell lineage, the analysis should be extended to the markers CD14 and CD48 on monocytes. Furthermore, we advise a serial monitoring for PNH patients as well as for patients with bone marrow 
failure syndromes without a present GPI-AP deficiency every 6 months or in case of significant clinical symptoms.

Recently, Richards et al. [29] showed that a relevant proportion of laboratories doing PNH testing by flow cytometry have significant problems with regard to falsepositive and false-negative results. On the other hand, due to the advent of a new targeted therapy option, early diagnosis and serial monitoring of $\mathrm{PNH}$ is gaining importance for a better patient management [17]. As a consequence, there is the urgent need for optimised flow cytometric protocols in PNH diagnosis. In conclusion, this analysis has demonstrated that the described flow cytometric method offers significant benefits in sensitivity and validity in PNH testing and can be therefore recommended for a wider use.

Acknowledgments The authors would like to thank Gisela Baur, Thomas Becker, and Rosi Leichtle for skilful technical and statistical evaluation assistance.

Authorship and disclosures $\mathrm{BH}$ and HS took primary responsibility for the paper and designed research. BH and MR performed laboratory work. $\mathrm{BH}, \mathrm{HS}$, and MR analysed the data and wrote the paper.

$\mathrm{BH}$ and HS were advisors for and received honoria from Alexion. MR reported no potential conflicts of interest.

Open Access This article is distributed under the terms of the Creative Commons Attribution Noncommercial License which permits any noncommercial use, distribution, and reproduction in any medium, provided the original author(s) and source are credited.

\section{References}

1. Dacie JV (1963) Paroxysmal nocturnal haemoglobinuria. Proc R Soc Med 56:587-596

2. Oni SB, Osunkoya BO, Luzzatto L (1970) Paroxysmal nocturnal hemoglobinuria: evidence of monoclonal origin of abnormal red cells. Blood 36:145-152

3. Myata T, Takeda J, Iida Y, Yamada N, Inoue N, Takahashi M, Maeda K, Kitani T, Kinoshita T (1993) The cloning of PIG-A, a component in the early step of GPI-anchor biosynthesis. Science 259:1318-1320

4. Mortazavi Y, Merk B, McIntosh J, Marsh JC, Schrezenmeier H, Rutherford TR, BIOMED II Pathophysiology and Treatment of Aplastic Anaemia Study Group (2003) The spectrum of PIG-A mutations in aplastic anemia/paroxysmal nocturnal hemoglobinuria: a high incidence of multiple mutations and evidence of a mutational hot spot. Blood 101(7):2833-2841

5. Bessler M, Mason PJ, Hillmen P, Miyata T, Yamada N, Takeda N, Luzzatto L, Kinoshita T (1994) Paroxysmal nocturnal hemoglobinuria is caused by somatic mutations in the PIG-A gene. EMBO J 13:110-117

6. Hillmen P, Lewis SM, Bessler M, Luzzatto L, Dacie JV (1995) Natural history of paroxysmal nocturnal hemoglobinuria. N Eng J 333:1253-1258

7. Hillmen P, Hows JM, Luzzatto L (1992) Two distinct patterns of glycosylphosphatidylinositol (GPI) linked protein deficiency in the red cells of patients with paroxysmal nocturnal haemoglobinuria. Br J Haematol 80(3):399-405

8. de Latour RP, Mary JY, Salanoubat C, Terriou L, Etienne G, Mohty M, Roth S, de Guibert S, Maury S, Cahn JY, Socie G (2008) Paroxysmal nocturnal hemoglobinuria: natural history of disease subcategories. Blood 112:3099-3106

9. Hernandez-Campo PM, Almeida J, Acevedo MJ, Sanchez ML, Alberca I, Vidriales B, Martinez E, Romero JR, Orfao A (2008) Detailed immunophenotypic characterization of different major and minor subsets of peripheral blood cells in patients with paroxysmal nocturnal hemoglobinuria. Transfusion 48:1403-1414

10. Richards SJ, Hillmen P (2002) Immunophenotypic analysis of PNH cells. Curr Protoc Cytom, Chapter 6:Unit 6.11

11. Nebe T, Schubert J, Gutensohn K, Schrezenmeier H (2003) Flow cytometric analysis of GPI-deficient cells for the diagnosis of paroxysmal nocturnal hemoglobinuria (PNH). J Lab Med 27:257265

12. Schrezenmeier H, Hildebrand A, Rojewski M, Hacker H, Heimpel H, Raghavachar A (2000) Paroxysmal nocturnal haemoglobinuria: a replacement of haematopoietic tissue? Acta Haematol 103:41-48

13. Moyo VM, Mukhina GL, Garrett ES, Brodsky RA (2004) Natural history of paroxysmal nocturnal haemoglobinuria using modern diagnostic assays. Br J Haematol 126:133-138

14. Richards SJ, Barnett D (2007) The role of flow cytometry in the diagnosis of PNH in the clinical laboratory. Clin Lab Med 27:577-590

15. Hall C, Richards S, Hillmen P (2003) Primary prophylaxis with warfarin prevents thrombosis in PNH. Blood 102:3587-3591

16. Hillmen P, Muus P, Dührsen U, Risitano A, Schubert J, Luzzatto L, Schrezenmeier H, Jeffrey S, Brodsky RA, Hill A, Socie G, Bessler M, Rollins S, Bell L, Rother R, Young NS (2007) Effect of the complement inhibitor eculizumab on thromboembolism in patients with paroxysman nocturnal hemoglobinuria. Blood 110 (12):4123-4128

17. Schrezenmeier H, Höchsmann B (2009) Eculizumab opens a new era of treatment for paroxysmal nocturnal hemoglobinuria. Expert Rev Hematol 2(1):7-16

18. Hill A, Richards SJ, Hillmen P (2007) Recent developments in the understanding and management of PNH. Br J Haematol 137:181192

19. Hill A, Hillmen P, Richards SJ et al (2005) Sustained response and long term safety of eculizumab in PNH. Blood 106:2559 2565

20. Schrezenmeier H, Hertenstein B, Wagner B et al (1995) A pathogenetic link between aplastic anemia and paroxysmal nocturnal haemoglobinuria is suggested by a high frequency of aplastic anemia patients with a deficiency of phophatidylinositol glycan anchored proteins. Exp Hematol 23:81-87

21. Parker C, Omine M, Socie G et al (2005) Diagnosis and management of paroxysmal nocturnal hemoglobinuria. Blood 106(12):3699-3709

22. Hall SE, Rosse WF (1996) The use of monoclonal antibodies and flow cytometry in the diagnosis of paroxysmal nocturnal hemoglobinuria. Blood 87:5332-5340

23. Brodsky RA, Mukhina GL, Li S et al (2000) Improved detection and characterization of paroxysmal nocturnal hemoglobinuria using fluorescent aerolysin. Am J Clin Pathol 114:459-466

24. Rosse WF, Dacie JV (1966) Immune lysis of normal human and paroxysmal nocturnal hemoglobinuria $(\mathrm{PNH})$ red blood cells. J Clin Invest 45(5):736-745

25. Navenot JM, Muller JY, Blanchard D (1998) Investigation of the survival of paroxysmal nocturnal hemoglobinuria red cells through the immunophenotyping of reticulocytes. Transfusion 38 (4):337-342

26. Taylor VC, Sims M, Brett S, Field MC (1997) Antibody selection against CD52 produces a paroxysmal nocturnal haemoglobinuria 
phenotype in lymphocytes by a novel mechanism. Biochem $\mathrm{J}$ 322:919-925

27. Young NS (1992) The problem of clonality in aplastic anemia: Dr Dameshek's riddle, restated. Blood 79(6):1385-1392

28. Bessler M, Mason P, Hillmen P, Luzzatto L (1994) Somatic mutations and cellular selection in paroxysmal nocturnal haemoglobinuria. Lancet 343(8903):951-953

29. Richards SJ, Whitby L, Cullen MJ, Barnett D et al (2009) Development and evaluation of a stabilized whole-blood preparation as a process control material for screening of paroxysmal nocturnal hemoglobinuria by flow cytometry. Cytom B Clin Cytom 76 B:47-55

30. Wang SA, Pozdnyakova O, Jorgensen JL, Medelros J, Stachurski D, Anderson M, Raza A, Woda BA (2009) Detection of paroxysmal nocturnal hemoglobinuria clones in patients with myelodysplastic syndromes and related bone marrow diseases, with emphasis on diagnostic pitfalls and caveats. Haematologica 94(1):29-37

31. Helley D, de Latour RP, Porcher R, Rodrigues CA, Galy-Fauroux I, Matheron J, Duval A, Schved JF, Fischer AM, Socié G, French Society of Hematology (2010) Evaluation of hemostasis and endothelial function in patients with paroxysmal nocturnal hemoglobinuria receiving eculizumab. Haematologica 95(4):574-581

32. Hill A, Rother RP, Wang X, Morris SM Jr, Quinn-Senger K, Kelly R, Richards SJ, Bessler M, Bell L, Hillmen P, Gladwin MT (2010) Effect of eculizumab on haemolysis-associated nitric oxide depletion, dyspnoea, and measures of pulmonary hypertension in patients with paroxysmal nocturnal haemoglobinuria. $\mathrm{Br} \mathrm{J}$ Haematol 149(3):414-425 